\title{
USING LEARNING BY DOING METHODOLOGY FOR TEACHING MULTI-AGENT SYSTEMS
}

\author{
J. Palanca, J. Jordán, V. Julián \\ Universitat Politècnica de València (SPAIN)
}

\begin{abstract}
In recent years the teaching of subjects related to Artificial Intelligence has grown notably in higher education degrees. This is the case of the discipline of multi-agent systems, which usually is part of the majority of master's degrees in Artificial Intelligence. Multi-agent systems (MAS) offer solutions for distributed decision making, where a set of autonomous intelligent agents must reach an agreement to solve a problem. These types of problems are usually complex and distributed, difficult to abstract and simplify for classroom teaching. The main problem that teachers of this subject have to face, is to be able to integrate the whole set of related techniques and algorithms in a practical example that is easy to understand and address within the framework of the planning of a course.
\end{abstract}

This paper deals with the use of the "learning by doing" methodology in a subject of multi-agent systems in the Master's Degree in Artificial Intelligence at the Universitat Politècnica de València. This methodology is applied by avoiding master classes to focus on practice. The classes become a scientific-technological experience. The students and the teacher are a team working with a common purpose, seeking to achieve a goal.

To do this, the whole course has been reformulated, proposing the students to solve different typical problems of the MAS area on the same domain, in this case the improvement of urban mobility and the efficient use of energy in the cities. It is considered to be a sufficiently current topic that can motivate the student to participate and propose solutions.

To achieve this objective, a multi-agent system tool has been developed that allows students to simulate the different situations proposed and develop solutions. The tool provides them with an urban simulation environment where they can easily introduce their own strategies to be carried out by each simulation agent. In this way, students are proposed different challenges where they can develop negotiation strategies to simulate the operation of urban taxi fleets, and cooperation strategies, where different agents help each other to achieve a common goal.

This tool, called SimFleet, has been developed in an open way and published as open source, so that it can be used by any teaching team that wishes to do so, and even receive external contributions and improvements thanks to its open character.

This learning by doing methodology supported by the SimFleet simulation tool has been applied in two consecutive academic years obtaining better results in student assessment and learning than in previous courses. Furthermore, the results of the student satisfaction surveys have shown a notable increase when using these technologies, which reinforces the idea that this type of learning is more useful and more satisfactory for students.

Keywords: Artificial intelligence, multi-agent system, learning by doing, simulation.

\section{INTRODUCTION}

Teaching artificial intelligence courses involves learning lots of algorithms but also needs to practice a lot to achieve the desired skills in the subject. To this end, in the subject of multi-agent systems in the Master's Degree in Artificial Intelligence at the Universitat Politècnica de València the authors have considered how best to practice and learn the techniques of artificial intelligence (and specifically intelligent agents and multi-agent systems [1][2]) taught in the course.

Multi-agent systems (MAS) are an area of artificial intelligence that covers different aspects related to distribution in decision making, cooperation or conflict resolution. These concepts are difficult for students to understand without a strong practical component. A clear example is to design and develop a complete negotiation process between different intelligent agents where the students may experience and test different strategies during the negotiation in a real and easily understandable domain. In order to facilitate the students' learning process, it is mandatory to have an appropriate 
methodology and a practical tool that simplifies the development of systems of this kind and it allows an incremental practical learning during the whole course.

According to this, a methodology for learning these skills is presented in this paper. The methodology is focused on the learning-by-doing principle, and the software that has been specifically designed and developed for this course is also presented. This software, called SimFleet, presents a simulation environment where it is possible to develop and propose different challenges that are introduced to the students in order to apply the knowledge about intelligent and multi-agent systems in running cases.

\section{METHODOLOGY}

In this section we present the learning-by-doing methodology and how it has been applied to the context of teaching multi-agent systems in the Master's Degree in Artificial Intelligence. In addition, the details of the SimFleet tool that has been used for this purpose are also explained.

\subsection{Learning by Doing}

The learning-by-doing methodology is based on the learning of the students from direct experience [3][4]. This methodology was first developed by John Dewey [5]. It can also be called experiential learning [6], and more specifically, it would be 'learning through reflection on doing'. In this way, students take an active role and learn through experience and subsequent reflection on what they are doing.

This methodology has been widely studied and applied, even Aristotle in 350 B.C. affirmed "for the things we have to learn before we can do them, we learn by doing them" [7], since it is one of the most common forms of human learning.

In an engineering context, its application makes a lot of sense since it can help to better understand the concepts that students must learn. Furthermore, it can also be combined with theoretical classes in which students have a passive role in learning the basics of some knowledge, and then move into action and put that knowledge into practice to achieve its understanding and full development.

Therefore, to teach students the main concepts of multi-agent systems, the use of this methodology is proposed using the SimFleet simulator as an environment for programming and experimentation. This simulator is a multi-agent system that has elements of urban fleets represented as agents, a communication system between agents, and the behaviours that define the actions carried out by these agents. The main characteristics of SimFleet are discussed in Section 2.2.

\subsection{SimFleet}

SimFleet [8] is a software developed by the authors to allow students to learn artificial intelligence capabilities by means of the learning-by-doing methodology. It provides a development interface that has a graphical frontend to show the simulation in a pretty way (Fig. 1) and a backend where it is easy to introduce new behaviours to be tested. The goal of SimFleet is to simulate open fleets of vehicles and to develop intelligent coordination and negotiation algorithms to manage different situations with open fleets (e.g., assign the closest taxi, distribute the fleet around the city equally, or to carry out an auction to get a client, among others).

SimFleet is built over a multi-agent system called SPADE [9], and the students use multi-agent algorithms to solve the challenges proposed by the teacher, and in the meantime improving their skills in artificial intelligence.

The approach that SimFleet uses to help teach these skills is to provide a fully functional simulation environment as an open world where the students have some pre-defined spaces to include their solutions. To this end, SimFleet makes use of a design pattern called Strategy. Design patterns are solutions to common problems in the design of software. Each pattern is like a blueprint that can be customized to solve a problem of particular design of your code. Strategy is a behavioural design pattern that allows you to define a family of algorithms, place each of them in a separate class and make their objects interchangeable. This means that students can work in a separate file, where they write their negotiation or coordination strategies (e.g., negotiating the closest taxi) and just inject this file into SimFleet and see how it works.

SimFleet is composed of three types of agents: drivers, customers, and fleet managers. Students are asked to design the strategy for one or more of these agent types to solve a challenge. Following the 
example of locating the nearest taxi, students should create, for example, a customer behaviour that asks fleet managers for a taxi for their position. The fleet managers would ask their taxis which one is free and closest to the customer and put them in contact. Finally, the driver and the customer would negotiate a price for the trip and if they reached an agreement the trip would take place.

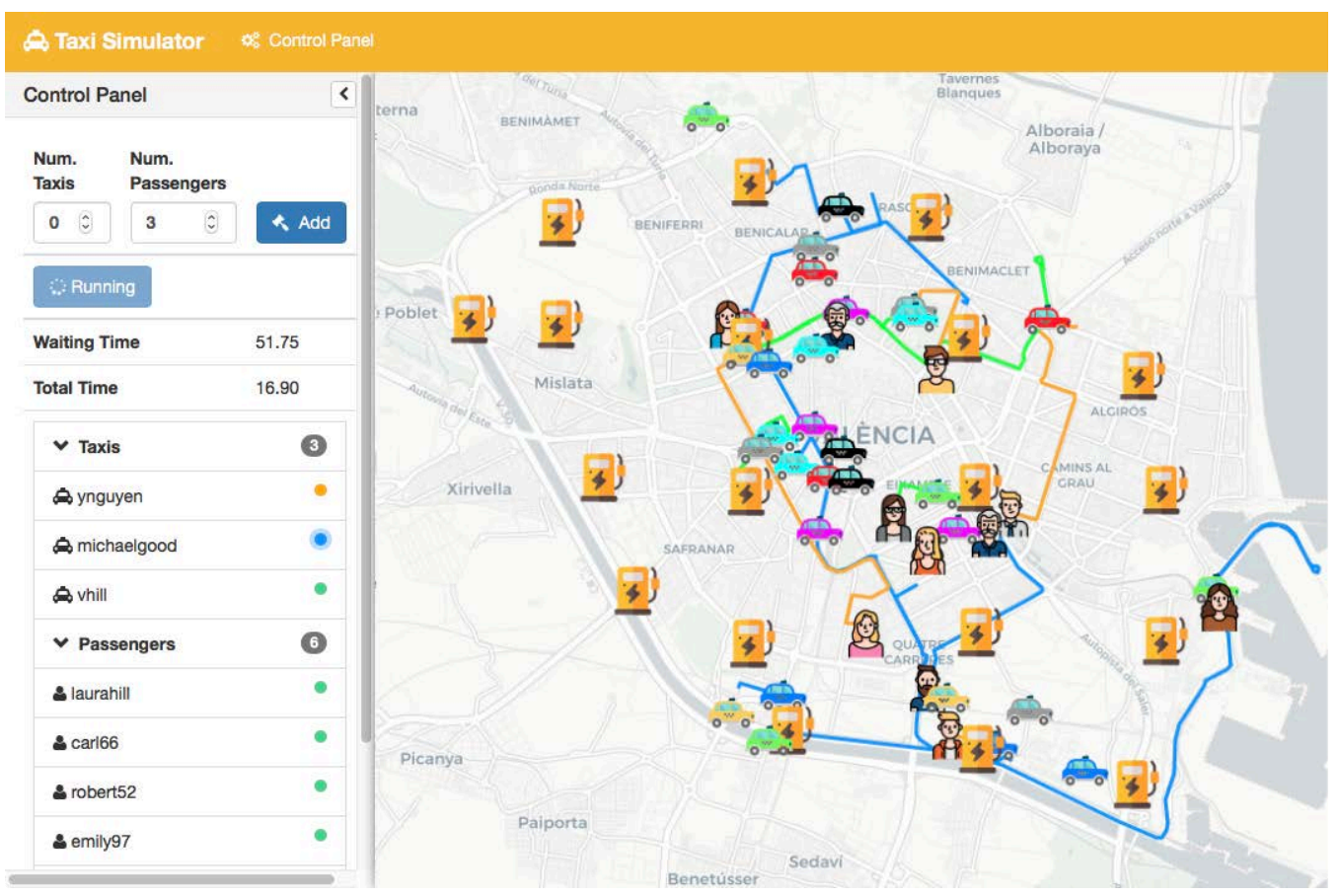

Figure 1. Graphical frontend of SimFleet.

Since SimFleet is a multi-agent application, this kind of problem can be solved on a big scale, with thousands of agents (i.e., taxis, customers, etc.) working in a city. SimFleet collects statistics of each simulation (e.g., the number of finished trips, the distance covered by the vehicles or the time that customers were waiting for a vehicle) to evaluate and compare the solutions proposed by each student.

In order to propose more advanced challenges to the students there is a new type of agent, called station, that represents static agents that cannot be moved. This type of agent is used to create challenges where drivers or customers interact with taxi or bus stations, electric charging stations (in the case of electric vehicles) or gas stations, among others. These station agents introduce more complex and interesting problems to be proposed to students. Station agents can interact with other agents and manage waiting queues, negotiate with other agents, etc. Some of the challenges that have been proposed to the students include planning routes considering the need of charging the electric vehicle in route or distribute taxis in all stations of a city taking into account the forecast of the demand.

\subsection{Application of the Learning-by-doing Methodology to SimFleet}

In this section we discuss in detail how the learning-by-doing methodology is applied using SimFleet in the subject of multi-agent systems in the Master's in Artificial Intelligence. In general terms, students get to know and work on different parts of SimFleet in an incremental manner and thus learn the concepts of MAS.

In the first session of the course, the tool is presented giving the necessary details for its global understanding. In addition, the students already start to use the tool directly and can thus test the most basic functionalities with examples that are already prepared and hardly require any knowledge to make them work.

Later, the students learn how the agents are implemented in the tool. In this way, the concept of agent is introduced, and students can test agents already implemented within the tool and see the differences between the different agents. 
Then, it is explained how communication is carried out in MAS. For this purpose, the students begin to see the implementation and demonstration of the exchange of messages between the agents. In addition, they also have a web visualization of the exchange of messages between the agents in real time, so they can see what each specific agent sends and receives.

As communication is a fundamental part of MAS, students should also implement some examples of the "query" and "request" protocols. Regarding the "query" protocol [10], students should implement an example of it where a taxi driver asks if there is space available at a gas station. Regarding the "request" protocol [11], the example to be implemented by the students is that a customer requests a taxi to take him from his point of origin to his destination. Both protocols require implementation both in the agent that issues the request/query and in the agent that receives and responds to it. These practices ensure that the student understands the implications of communication in MAS and sees the importance of the communication protocols, as well as the entire message flow involved.

Another of the fundamentals of MAS is the coordination of agents. Therefore, students must implement the contract net protocol [12] which consists of a manager who looks for other agents who are capable of doing a task. Then, after the manager's initial call, the interested agents can make their proposals for carrying out the task. The manager chooses the offer that best suits him/her and informs the agent. Finally, when the agent with the assigned task has finished carrying it out, he communicates it to the manager, thus ending the protocol. The implementation of this complex protocol helps students to understand coordination in MAS.

The last topic dealt with by MAS students in this Master's course is negotiation. To this end, it is proposed that they implement an auction mechanism. An auction in MAS involves a system in which agents can make their bids to obtain a product or a contract and requires a whole coordination system so that the final allocation of the auction can be computed. These concepts help students to go deeper into the concepts of behaviour, communication, and coordination of agents, in addition to seeing the importance of MAS for other related fields such as negotiation.

\section{RESULTS}

The new methodology has been tested during the last two years in the previously mentioned master's course. The course about "Multi-agent Systems" is part of the courses taught at the Master's Degree in Artificial Intelligence at the Universitat Politècnica de València. The subject has about 30 students who were proposed at the beginning of the course to perform the tasks discussed in the previous section.

At the end of each academic year, a survey was conducted to determine the degree of student satisfaction with the methodology and use of the tool and the activities related to it. The survey included 9 questions about how their experience and satisfaction with the course was. Out of the 67 students (33 and 34 in each academic year) which participated in the activities, 51 responded to the survey (a $76 \%$ of participation).

Results show, in general, that the students have a favourable opinion of the use of the proposed methodology. Fig. 2 presents the degree of usefulness perceived by students after experimenting with SimFleet during the proposed activities, in relation to understanding four of the key concepts introduced in the course:

- C1: communication among agents, that is, how agents communicate with each other in a MAS.

- C2: communication protocols, that is, how communication protocols are defined and used by agents in order to communicate effectively.

- C3: coordination in MAS, related with strategies used by agents to coordinate between them in order to achieve collective goals.

- C4: negotiation in MAS, covering the way some typical MAS establish negotiation protocols such as the basic contract net protocol and some kind of auctions. 


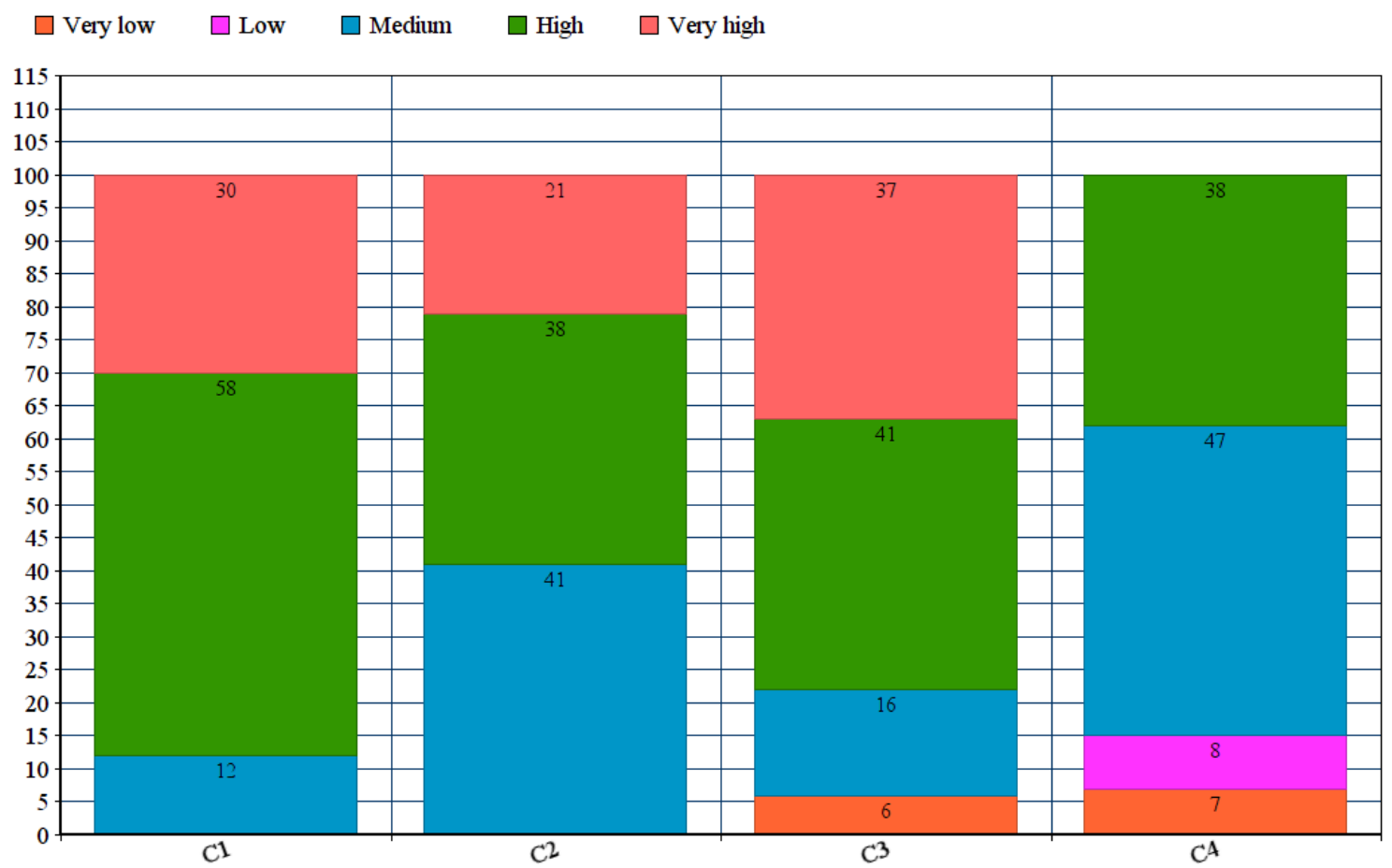

Figure 2. Usefulness of the proposed methodology using SimFleet.

The figure includes a graph with four columns, where each column represents each of the key concepts explained. The accumulated percentage of perceived usefulness is shown for each concept. The students were asked to value their answers for each question in a range that goes from very low to very high. Analysing the results in detail, students considered very useful the methodology to understand the concept of communication among agents, with $88 \%$ of answers high or very high, and mostly useful in the concept of communication protocols in MAS, with $59 \%$ of answers high or very high, and $41 \%$ medium.

In the case of coordination in MAS, the general opinion is favourable. A $78 \%$ of answers were high or very high, but it had a $6 \%$ of very low answers. Regarding the last concept, negotiation in MAS obtained the worst result, although it was still quite satisfactory, $0 \%$ of the students responded very high and $15 \%$ responded low or very low. Nevertheless, most of the students answered high $(38 \%)$ or medium (47\%).

\section{CONCLUSIONS}

This paper has presented the use of the learning-by-doing methodology to teach the main concepts of multi-agent systems in the Master's Degree in Artificial Intelligence at the Universitat Politècnica de València. The advantage of this methodology is that students learn the concepts better by working directly on them through experimentation. To this end, the SimFleet tool has been used, which is an urban fleet simulator built with multi-agent systems technology.

The results of student satisfaction obtained through surveys as well as the grades of the evaluation of the students' knowledge have been significantly better since the application of this methodology in the last two academic years.

Finally, one line of future work to be developed in future courses is the inclusion of team formation in different types of fleets. This would allow students to go deeper into coordination in multi-agent systems since the formation of teams representing different types of urban fleets is a current challenge. 


\section{ACKNOWLEDGEMENTS}

This work was partially supported by MINECO/FEDER RTI2018-095390-B-C31 project of the Spanish government.

\section{REFERENCES}

[1] M. Wooldridge, An introduction to multiagent systems, John Wiley \& Sons, 2009.

[2] M. Wooldridge, N. Jennings, "Intelligent agents: Theory and practice", The knowledge engineering review, 10 (2), 115-152, 1995.

[3] H. W. Reese, "The learning-by-doing principle", Behavioral Development Bulletin, 17(1), 1-19, 2011.

[4] L. Bot, PB. Gossiaux, CP. Rauch, and S. Tabiou, "'Learning by doing': a teaching method for active learning in scientific graduate education", European Journal of Engineering Education, 30:1, 105-119, 2005.

[5] J. Dewey, "Experience and education", The Educational Forum 50 (3), 241-252, 1986.

[6] C. Beard, The Experiential Learning Toolkit: Blending Practice with Concepts, Kogan Page Publishers, 2010.

[7] Aristotle. Nicomachean Ethics, Book II. 350 B.C. Accessed 12 January, 2021, http://classics.mit.edu/Aristotle/nicomachaen.2.ii.html

[8] J. Palanca, A. Terrasa, C. Carrascosa, V. Julián, "SimFleet: A New Transport Fleet Simulator Based on MAS", Highlights of Practical Applications of Survivable Agents and Multi-Agent Systems, The PAAMS Collection, PAAMS 2019, Communications in Computer and Information Science, vol 1047. Springer, Cham, 2019.

[9] J. Palanca, A. Terrasa, V. Julián, C. Carrascosa, "SPADE 3: Supporting the New Generation of Multi-Agent Systems", IEEE Access 8, 182537-182549, 2020.

[10] FIPA query protocol, Accessed 12 January, 2021. Retrieved from http://www.fipa.org/specs/fipa00027/SC00027H.html.

[11] FIPA request protocol, Accessed 12 January, 2021. Retrieved from http://www.fipa.org/specs/fipa00026/SC00026H.html.

[12] R. Smith, "The Contract Net Protocol: High-Level Communication and Control in a Distributed Problem Solver", IEEE Transactions on Computers, C-29 (12): 1104-1113, 1980. 